anderen Alkalisalzen, (von denen ja bekanntlich Salpeter auch eine Fïbrin lösende Wirkung hat) oder bei heftiger Entzündung direct antiphlogistische Mittel, wie Eis, oft vielleicht mehr Nutzen, immer aber gewiss weniger Schaden stiften. - -

Eine Anzahl von Fällen, welche der hier gegebenen Darstellung zu Grunde liegt, habe ich auf der Klinik des Herrn Geheimrath Traube beobachtet, welchem ich auch an dieser stelle meinen besten Dank abzustatten, nicht unterlasse.

\title{
VI.
}

Ileterotopie grauer Hirnsubstanz im Markstamme der Hemisphären des kleinen Gehirns. Drei Beobachtungen.

\author{
Von Dr. Franz Meschede, \\ zweitem Arzte der Westpreussisclien Provinzial-Kranken-Anstalten zu Schwelz.
}

(Hierzu Taf. II. Fig. 3, 4, 8 u. 9.)

Auf der 42. Versammlung deutscher Naturforscher und Aerzte zu Dresden im Jahre 1868 habe ich über eine bis dahin noch nicht beobachtete Abnormität des kleinen Gehirns, nehmlich über Heterotopie grauer Hirnsubstanzim Markstamme der einen Hemisphäre des kleinen Gehirns eine kurze Mittheilung gemacht und die patbologisch-anatomischen Verhältnisse derselben unter Vorzeigung photographischer und anderer Abbildungen iu der zweiten Sitzung der psychiatrischen Section rom 19. September 1868 demonstrirt (Tageblatt No. 5, S. 84).

Seitdem ist diese Veränderung noch in $z$ wei weiteren Fällen von mir gefunden worden.

Da über diese Anomalie des kleinen Gehirns Beobachtungen anderer Autoren nicht vorzuliegen scheinen, mir wenigstens solche auch seit meiner eben erwähnten Mittheilung nicht bekannt geworden sind, - andererseits aber auch die erwähnte Abnormität nicht ohne alle Bedeutung für die Pathologie des Gehirns sein dürfte, so habe ich es für angezeigt gehalten, sowohl von dem vorerwähnten, vorläufig notificirten, Falle eine etwas genauere Beschreibung als auch 
von den beiden anderen eine kurze, das Wesentlichste der Krankhejtsgeschichte und der Autopsie entbaltende Mittheilung hier zu veröffentlichen - zumal gerade vom kleinen Gehirn pathologischanatomische Veränderungen verhältnissmässig selten und überhaupt wenig bekannt sind.

\section{Erste Beabachtung.}

Die in Rede stehende Anomalie, Heterotopie grauer Hirnsubstanz im Markstamme des kleinen Gehirns, wurde von mir zuerst im Januar 1868 bei der Section einer epileptischen Geisteskranken vorgefunden - und zwar in der linken Kleinhirnbemisphäre, im peripheriscben Theile des Markstammes des Arbor vitae. Es repräsentirte sich dieselbe auf einem parallel der Längsaxe des verlängerten Markes und senkrecht zur Horizontalebene durch die genannte Hemisphüre geführten Durchschnitte als ein Streifen röthlich-grauer Hirnsubstanz von etwas unregelmässiger Form und von circa 13 bis $15 \mathrm{Mm}$. Länge und $\frac{1}{2}$ bis $3 \mathrm{Mm}$. Breite, welcher längs der unteren Grenze des in seiner Lăngsrichtung durchschnittenen Stammes des Arbor vitae in leicht wellig gebogenem Verlaufe in der Richtung von vorn nach hinten sich erstreckte, mit der grauen Substanz der benachbarten Windungen nirgends einen Z usammenhang zeigte, vielmehr nach allen Seiten hin von weisser Substanz umgeben war. An einzelnen Stellen trat dieser graue Streifen sebr nahe an die graue Substanz der zunächst gelegenen Windungen heran, doch war auch an diesen Stellen zwischen beiden noch eine schmale Zone Medullarsubstanz mit aller Deutlichkeit zu constatiren. Auch andere in verschiedenen Richtungen geführte Durchschnitte liessen nirgends einen Zusammenhang mit der grauen Substanz der Windungen erkennen. Diese Durchschnitte ergaben ferner, dass die Mächtigkeit der heterotopischen grauen Masse in der Richtung nach aussen allmählich abnahm, aber auch medianwärts keine stärkere Entwickelung erreichte und dass die kürzeste Entfernung zwischen ihr und der grauröthlichen Grenzlamelle des Nucleus dentatus cerebelii noch $7 \mathrm{Mm}$. betrug.

Zum Behuf einer möglichst naturgetrenen Darstellung der Lageverhältnisse und der Configuration der Heterotopie babe ich, so gut es sich bei der verbältnissmässig weichen Beschaffenheit der Hirnsubstanz thun liess, eine pbotographis che Abbildung eines in der oben bezeichneten Richtung geführten Durebschnitts aufnehmen lassen; nach derselben ist die Fig. 3 auf Taf. II in Lithographie ausgeführt. Man sieht auf dieser Abbildung deutlich den Verlauf der heterotopischen Masse, welche als grauer Streifen erscheint, hauptsächlich in seinem hinteren Abschnitte entwickelt ist und sich hier noch ziemlich weit bis in den einen (unteren) der beiden terminalen Hauptäste erstreckt, in welche sich der Stamm des Arbor vitae spaltet.

Die mikroskopische Untersuchung der fraglichen grauen Masse liess in derselben viele grosse, deutlich als Ganglienzellen charakterisirte, pigmentlose Zellen erkennen. Dieselben stimmten mit den in der grauen Corticalsubstanz der Windungen vorkommenden durcbaus überein. Sie zeigten jm Allgemeinep die Beschaffenheit, Form und Grössenverhältnisse der von kölliker abgebildeten Zellen 
der grauen Rindenschicbt des kleinen Gehirns, nur dass der eine zarte Fortsatz nicht mit voller Bestimmtheit nachzuweisen war, wahrscheinlich aus dem Grunde, weil die Hirnsubstanz ödematös und dadurch eine grössere Brüchigkeit der zarteren Fortsätze bedingt war, erbärtende Chemikalien aber absichtlich nicht in Anwendung gezogen worden waren, um nicht artificiell pseudopathologische Veränderungen herbeizuführen. An einzelnen Zellenformen konnte man deutlich die Stellen erkennen, wo breite Fortsätze sich rom Zellenkörper abgetrennt hatten; an anderen dagegen scbienen Fortsätze ganz zu fehlen; die Identität der Zellen mit wirklichen Ganglienzellen erwies sich übrigens schon auf den ersten Blick als unzweifelhaft. In Fig. 4 sind einige derselben bei circa 300 facher Vergrösserung abgebildet.

Ausser diesen grossen blassen Ganglienzellen mit feingranulirtem Inhalte fand sich eine feinkörnige Grondsubstanz, Nervenfaserzäge, sowie zahlreiche kernartige Körner, welche indess hier nicht so dicht vorhanden waren, wie die normalmässig in der graurötblicben Schicht der Windungen des klejuen Gehirns vorkommenden, - im Uebrigen aber diesen letzteren ziemlich ähnlich erschienen. $\mathrm{Z}$ wischen den Körnern zerstreut fanden sich endlich auch nocb kleine Zellen mit relativ grossen Kernen und zarter Membran in nicht geringer Anzahl vor.

Was die übrigen Sectionsergebnisse betrifft, so beschränke ich mich darauf, fólgende Punkte hervorzuheben:

Schädel leicht, ziemlich dünn, symmetrisch, mit deutlichen Nähten, Länge 18, grösste Breite $14 \mathrm{Cm}$. Dura mater mit dem Schädel ziemlich fest verwachsen, die weicben Häute ödematös, aber ohne erhebliche Trübung. Oedem der subarachnoidealen Räume. Bedeutende Hyperämie der Gefässe an der convexen Oberfläche des grossen Gehirns. Olfactoril graufleckig. Hirnsubstanz, sowohl des grossen als auch des kleinen Gehirns ödematös und weich, Marksubstanz des Corp. dentat. cerebelli trübe und von verminderter Consistenz; innere Oberfläcbe des vierten Ventrikels und unterer Theil des verlängerten Marks ebenfalls abnorm weich, desgleichen in hohem Grade auch beide Acustici (hydropische Erweichung). In der rechten Hemispbäre des grossen Gebirns, in ejner der vorderen Windungen des hinteren Lappens eine Teleangiectasie ron etwa Linsengrösse. Seiten-Ventrikel mässig erweitert, mit klarem Serum gefüllt, Ependym weich. Corticalsubstanz beider Hemisphären aufallend weich und blass. Räckenmark: Perimedulläres Fettgewebe blutröthlich imbibirt, das Mark selbst bis etwa zur Höhe der 8. Rippe weich, im unteren Abschnitte von festerer Consistenz. Hintere und Seitenstränge des Rückenmarks auf dem Querdurchschnitt von tröbem, leicht in’s Graugelblicbe fallendem Anseben. Lungen: Emphysem, Hypostase; Spitze der rechten Lunge etwas angewachsen. Herz klein, fettlos; an der vorderen Oberfläche eine sehnige Verdickung.

Die vorstehend beschriebene Anomalie fand sich bei einer, wie schon erwähnt, epileptiscben Geisteskranken, Namens Draws, welche zum ersten Male in ihrem eilften Lebensjahre, unmittelbar nach Tanzen und wildem Umberiollen, epileptische Krämpfe 
bekommen hatte und seit dieser Zeit öfter, etwa alle Vierteljahre, späterhin etwa allmonatlich und in der letzten Zeit, kurz vor ihrer Aufnahme in die hiesjge Anstalt, fast alltäglich von epileptischen Anfällen heimgesucht worden war. Durch diese epileptischen Krampfanfälle scheinen Anfangs die geistigen Functionen nicht merklich gestört gewesen zu sein; denn erst später, als die D. etwa 23 Jahre alt war, machten sich auch Symptome von Gejstesstörung bei ibr geltend, indem sie unmittelbar nach einem epileptischen Anfalle in einen Zustand von Raserei und Geistesabwesenheit verfiel. Solche Anfälle wiederholten sich dann öfter und liessen bald die Unterbringung der D. in eine Irrenanstalt nothwendig erscheinen. Sie waren im Allgemeinen den bekannten Wuthanfällen der Epileptiker analog, zeichneten sich jedoch besonders durch einen hohen Grad von Unbesinnlichkeit und durch triebartige Agitation aus. Zur Zeit jener oft mehrere Tage lang sich hinziehenden Aufregungsparoxysmen erschien die Kranke fast ganz geistesabwesend und pflegte auf die ihr gestellten Fragen gar nicht oder doch nur durch einige wenig verständlich hingemurmelte Worte zu antworten, dagegen den Trieb zu bethätigen, um sich zu schlagen, zu beissen und davonzulaufen. Auf die Wuthparoxysmen folgte in der Regel ein komatöser Zustand, der nicht selten einige Tage anhielt. Abgeseben von der allgemeinen Umnebelung des Bewusstseins liessen die Sinnesorgane an und für sich sonst keine auffallenden Störungen wahrnehmen. - Als Kind soll die D. ziemlich still und "für sich" gewesen sein, in der Schule Fleiss, gutes Begriffsvermögen und sanften Charakter gezeigt, überbaupt soll es ihr an Verstand nicht gemangelt haben. Auch in Handarbeiten ist sie angeblich geschickt gewesen. Die Menstruation soll im dreizehnten Lebensjahre eingetreten und seitden sparsam, aber regelmässig gewesen sein.

In Bezug auf die Aetiologie dürften wohl noch folgende Punkte der Erwähnung werth sein:

Von sechs Geschwistern der Kranken sind angeblich fünf, nehmlich vier Schwestern und ein Bruder, vollkommen gesund; eine Schwester dagegen ist in ibrem dreiundzwanzigsten Lebensjabre von epileptischen Krämpfen befallen worden, demnächst aber, nachdem sie noch fünf Mal epileptỉsche Krämpfe gehabt, ganz frei von denselben geblieben. Von den beiden Eltern der D. war die Mutter älter als der Vater und bereits in die Jahre der Involutionsperiode, in die sogenannten Wechseljabre, eingetreten, - nebmlich, wenn die mir vorliegenden 
Zeitangaben richtig sind, bereits 45 bis $46 \mathrm{Jahre}$ alt, als sie ron der in Rede stehenden mit der beschriebenen Structur-Anomaliedes kleinen Gebirns bebafteten Tochter entbunden wurde. Der Vater, welcher Holzschläger war, soll $\mathbf{5} 7$ Jahre alt, an Phthisis gestorben sein.

\section{Zweite Beobachtung.}

(Vgl. Fig. 8 u. 9.)

Eine zweite, der vorstebend mitgetheilten in anatomischer Beziebung durchaus analoge Beobachtung batte ich im Jahre 1870 zu machen Gelegenbeit. Auch in diesem Fall war die Heterotopie nur in der einen Hemisphäre des kleinen Gehirns vorhanden und zwar war es auch hier gleichfalls wieder die linke Hemisphäre, welche die Abnormität darbot.

Eine weitere Anaiogie mit dem ersten Falle bot der Umstand, dass auch in diesem Falle der mit der fraglichen Anomalia cerebelli behaftete patient an schwerer, allerdings auf ganz anderer ätiologiseher Grundlage beruhender Epilepsie gelitten hatte.

Da der Fall auch in anderer Beziehung nicht ohne Interesse ist, so möge hier zunächst das Wesentlichste der Krankheitsgeschichte mitgetheilt sein:

F. Dkleija, Schäferknecht, geboren am 1. April 1834, seit seinem 19. Lebensjabre epileptisch, in die Irrenanstalt zu Schwetz aufgenommen den 12. Juni 1865, gestorben den 25. Juli 1870. Der Vater soll fröher getrunken haben, im Uebrigen aber Keinerlei erbliche Disposition nacbzuweisen sein. Kräftige Constitution, gutmäthiges Naturell, Vierzehn Jahre alt, und bis dahin angeblich immer gesund, gerieth U. mit seinem Kopfe in eine Häkselmaschine, und erlitt dabei eine so schwere Verletzung des Kopfes, dass er 14 Tage lang bewustlos und bis zu seiner vollständigen Wiederherstellung überhaupt 4 Monate lang in ärztlicher Behandlung gewesen sein soll. Nach mündlichen Mittheilungen der Angehörigen hat die Verletzung in einer perforirenden Schädelwande in der Schlafengegend bestanden; a uch Gebirnsubstanz soll sich aus der Wande entleert baben. Die Wunde verheilte unter Zurücklassung mebrerer Narben: eine über $9,2 \mathrm{Cm}$. lange befand sich etwas hinter der Linea semicircularis des Stimbeins und verlief, der letzteren ziemlich parallel, vom äussersten Ende der rechten Augenbraue beginnend bogenförmig nach hinten und oben bis in das Gebiet der behaarten Kopfhaut; etwa in der Mitte ihres Verlaufs war sie am tiefsten, über $0,5 \mathrm{Cm}$. tief und circa $1,4 \mathrm{Cm}$. breit; drei andere Narben von 1,4 bis 1,5 Cm. Länge befanden sich in der Gegend des rechten Augenwinkels, von letzterem nach verschiedenen Richtungen hin ausstrahlend.

Nach Heilung der Schädelwunde scheint U. etwa 4 Jahre hindarch von schweren Cerebralsymptomen frei geblieben zu sein; es wird nur berichtet, dass er etwa 2 Jahre später einmal eine Zeitlang wegen Kopfschmerzen bettlägerig gewesen sei, 
Etwa 4 Jabrenach der Kopfverletzung traten zuerst epileptische $\mathrm{Krä} \mathrm{mpfe}$ bei dem U. auf; dieselben machten sich in grösseren Zwischenräumen und in unregelmässigen Perioden wiederkehrend, noch eine geraume, nicht genauer bestimmte Zeit hindurch geltend, cessirten alsdann während eines Zeitraums von mehreren Jabren vollständig, um mit dem $2 \frac{1}{4}$ Lebensjahre von Neuem und mit grösserer Heftigkeit wieder a ufutreten. Unter dem mehrjährigen Einflusse des fallsücbtigen Zustandes machte sich allmăhlich auch eine Beeinträchtigung der psychischen Functionen geltend, anfangs nur protrahirte Unbesinnlichkeit, Gedächtnissschwäche, Mangel des practischen Interesses, Stumpfbeit und Gemüthsverstimmungen, - zuletzt auch Aufregungs- und Zornparoxysmen, die sich schliesslich zu furibunden Wuthanfällen der gefährlichsten Art steigerten und die nächste Veranlassung zur Unterbringung des U. in die hiesige Irrenanstalt gaben.

Der geistesgestörte Zustand, welchen $\mathbf{U}$. während seines hiesigen Aufenthaltes darbot, charakterisirte sich im Allgemeinen als epileptischer Blödsinn mit heftigen Anfällen blinder Wuth.

Die epileptischen Anfälle traten sawohl bei Tage als auch Nachts auf, vorwiegend jedoch am Tage; zumeist an mehreren auf einander folgenden oder doch nahe liegenden Tagen. So wurden im J. 1868 im Ganzen 101 epileptische Anfalle beobachtet, davon 64 am Tage und 37 Nachts; überhaupt wurden an 77 Tagen Krampfanfälle bei dem U. beobachtet und zwar an folgenden Tagen: 3., 14, 27., 28. Januar; 15., 23., 26., 27. Februar; 10., 14., 15., 24., 30., 31. Mărz; 1., 3., 5., 18., 25., 27., 28. April; 1., 2., 9., 10., 14., 19., 30. Mai; 4., 10 , 13., 18., 22., 23. Juni; 10., 19., 22., 25. Juli; 6., 7., 8., 9., 10., 15., 17. August; 1., 2., 3., 6., 7., 11. September; 1., 2., 5., 8., 10., 12., 13., 14., 17., 18., 19., 25., 27. October; 1., 2., 4., 5., 6., 7., 8., 9., 11., 13., 14., 26. November; 12., 17., 20. December. - Die einzelnen Anfälle erfolgten plötzlich obne vorhergehende Aura, waren immer von sehr kurzer Dauer und mit vollständiger Bewusstlosigkeit verbunden. Trat der epileptische Anfall ein, während der Kranke gerade auf dem Stahle sass, so pflegte dieser vornüber zu fallen; dagegen fiel er hinten über, wenn er im Steben von einem epileptischen Insulte betroffen wurde. Während des Krampfanfalls resp. während des soporösen Nachstadiums des epileptischen Insults machte sich häufig ein gleichsam automatisch wirkender Trieb z t m Zerreissen der eigenen Kleider bemerklich. Die Wuthanfälle pflegten sich nicht selten unmittelbar an den Krampfanfall selbst anzuschliessen. Von den intercurrent eintretenden Wuthanfällen abgesehen, war das Verhalten des Pat. sonst ein ganz apatbisch-blödsinniges.

Der Tod erfolgt in einem epileptischen Krampfanfalle am 25. Juli 1870.

Autopsie am 26. Juli. Schädel blutreich und schwer; Stirn- und Seitenwandbeine namentlich rechterseits stark verdickt: das rechte Stirnbein hat durchschnittlich eine Dicke von über $12 \mathrm{Mm}$, an der stärksten Stelle sogar eine solche von $14 \mathrm{Mm}$, während die Dicke des linken Stirnbeins an der entsprechenden Stelle nur $8 \mathrm{Mm}$. beträgt. Dicke des rechten Seitenwandbeins $12 \mathrm{Mm}$. Die Verdickung des Craniums ist hauptsächlich durch äbermässige Entwicklung der diploetischen Substanz bedingt, während die innere Tafel (mit Ausnahme des Bezirks der früheren Koochenverletzung) selır verdüpnt, papierdünn erscheint. Länge des 
Schädeldaches 17,7 Cm., grösste Breite 14,1 Cm., Breiten-Index sonach 79,66. In der r. Schläfengegend besteht ein ziemlich bedeutender Eindruck, welchem an der inneren Oberfläche des Craniums eine, etwa 1,5 Cm. gegen das Innere des Cavam Cranii vorgeschobene Vorwölbung entspricht; wie der borizontale Durchschnitt zeigt, ist letztere zum Theil hohl, insofern zwischen der inneren und äusseren Tafel mehrfache Lücken resp. unregelnässig gestaltete Hohlräume bestehen. Auf dem borizontalen Durchschnitte tritt ein eigenthümliches Lageverhältniss der früher fracturirten Partien des Craniums zu Tage, welcbes einigermaassen an die sogenannte Dislocatio ad longitudinem bei Fracturen mehr beweglicher Knochen erinnert, insofern nehmlich die hintere Partie des von dem Trauma betroffenen Schädelbezirks nach innen und auch etwas nach vorn verschoben erscheint, die vor der. Stelle der Continuitätstrennung gejegene Partie dagegen mit ihrer inneren Oberfläche auf der äusseren Oberfläche der ohengenannten Partie aufliegt, - entsprechend der Richtung der stattgehabten Schädelwunde von aussen und hinten nach innen und vorn. Diese Verschiebung ist allerdings zum grössten Theil nur eine $\mathrm{s} c \mathrm{~b}$ e in b a re und der Schein dadurch bedingt, dass durch $\mathrm{K}$ nochenneubildung sowohl zwischen den durch das Trauma, ausser Continuität gesetzten Partien der ingeren Knochentafel, als auch zwischen denjenigen der äusseren Knochentafel jederseits eine knöcherne Verbindung entstanden ist, welche an der äusseren Oberfläche vorwiegend als Fortsetzung des vorderen Bruchrandes, an der inneren 0berfläche dagegen hauptsächlich als Fortsetzung der inneren Tafel des hinteren Theils der fracturirten Schädelpartie erscheint, - dergestalt, dass beide Tafeln auf dem Durchschnitt an der Stelle der Verletzung eine ähnliche Lage zu einander haben und in ähnlicher Weise einen geschlossenen Raum umschliessen wie die beiden Curven eines Paragraphenzeichens (\$). Die stärkste Vorwölbung der Schädelwand nach innen entspricht gerade dem Verlaufe der rechten Art. meningea media; diederselbenentsprechende Knachenfurche fübrtgerade über einen steilen Hügel dieser Vorwölbung. Ein Theil der Schläfenbeinschuppe scheint ganz verloren gegangen und durch neugebildete Knochenmasse ersetzt zu sein. An dem vordersten Ende des Eindrucks besteht noch eine röbrenförmige, die Knochenwand in schiefer Richtung (von binten und aussen nach innen und vorn) durchsetzende, an der inneren und äusseren Oeffnung reichlich mit kleinen 0steophyten besetzte Perforation, Porencephalie, welche übrigens durch ein derbes fibröses Gewebe verschlossen ist. Der Körper (Felsentheil) des rechten Schläfenbeins ist stärker aufgewölbt als derjenige des linken. Gefässfurchen mässig stark entwickelt; Impressiones digit. an der Oberfläche des rechten Felsenbeins fast fehlend. Sin us stark angefüllt, D n $r$ a mat er etwas stärker als gewöbnlich mit dem Schädel verwachsen. Weiche Häute blutreich, etwas gotrübt und namentlich rechterseits merklich verdickt und auch etwas fester als gewöhnlich der Gebirnoberfläche anhaftend. Die Trübung und Verdickung ist besonders an der Basis ausgesprochen, jedoch auch auf der Convexität nicht unbedeutend.

An der Stelle des Eindrucks sind die entsprechenden Windungen mitsammt der Dura mater mit der eingedrückten knöchernen Schädelwand ziemlich fest verwachsen. Innerhalb der Hirnsubstanz der rechten Hemisphäre des grossen Gebirps befindet sich in dieser Gegend eipe etwa nussgrosse, mit klarem Serum 
gefüllte Cyste, welche einerseits ziemlich nahe der Oberfläche der Windungen sich befindet, andererseits bis dicht an die Wandung des absteigenden Horns des recbten Seitenventrikels reicht. Die zwischen dem Ventrikel resp. dessen Born und der in Rede stebenden Cyste befindliche Partie Gehirnsubstanz ist zum Theil grau degenerirt. Die innere Wandung der Cyste erscheint unregelmässig, mit spinnwebenartigen Fortsätzen versehen. Die Gefässplexus der Ventrikel mit cystoiden Bildungen besetzt. - Medullarsubstanz des grossen Gehirns übermässig blutreich, von trübem Ausseben. Innere Lage der Corticalsubstanz in den Windungen der Convexität in ziemlich hohem Grade degenerirt, gelblich entfärbt, stellenweise atrophisch; in den Windungen anderer Bezirke ist diese Veränderng weniger ansgesprochen. - Der rechte Temporal-Lappen kleiner als der linke und etwas weicher als normal: Windungen zum Theil atrophisch und auf dem Durchschnitte eine schmutzig graugelbe Färbung darbietend.

Gehirnnerven obne erhebliche Anomalien; nur an den Wurzeln der Nervi vagi und Glossopharyngei kleine weisse maulbeerförmige senfkorngrosse Knötchen.

Cerebellam: Arachnoidea im Umfange des kleinen Gehirns nur in geringem Grade getrübt. - In der linken Hemisphäre des kleinen Gebirns befindet sich im Markstamme eine Einlagerung grauer Hirnsubstanz. Auf einem in derselben Richtung wie in dem vorstehend mitgetheilten Falle durch die linke Hemispbäre geführten Durchschnitte erscheint dieselbe als ein circa 5 bis $6 \mathrm{Mm}$. langer und eirca $1 \mathrm{Mm}$. breiter, rings von medullarer Hirnsubstan umgebener grauröthlicher Streifen von dem Ausehen grauer Hirnsubstanz, welcher ziemlich genau an derselben Stelle sich befindet, an welcher auch die vorstehend bereits beschriebene Hetęrotopie vorbanden war, nehmlich längs der unteren Grenzedes Markstammes, da wo sich von dem Hauptamme die einzelnen Aeste für die verschiedenen Lappenderunteren 0 berf 1 äche der Kleinhirn-Hemisphärenabzeigen. Durchschritte, die in verschiedenen Richtungen durch die Substanz der linken Hemispbäre geführt wurden, ergaben nirgends einen Zusammenhang der heterotopischen grauen Masse mit der grauen Corticalschicht der Windungen.

Brustböble: Rechte Lunge tuberculös, Herz ziemlich stark mit Fett bewachsen.

Ba uchhöhle: Fettleber. Gallenblase. mit dickflüssiger und dankelgefärbter Galle gefullt, ansserdem 96 kleine, durebschnittlich erbsengrosse Gallensteine enthaltend; letztere hatten eine gelbliche nahezu erbsengelbe Färbung und eine unregelmässig polyëdrisch abgeplattete oberfläche.

Die mikroskopische Untersuchung der fraglichen grauen Masse liess in derselben zahlreiche grosse Nervenzellen erkennen; dieselben zeigten sich in verschiedenen formen, waren theils von annähernd ovalairer Form, theils mehr spindel- oder kolbenförmig, feingranulirt, ohne eigentliches Pigment und mit starken breiten Fortsätzen versehen. Sie erwiesen, sicb durch Form, Grösse, Färbung, Beschaffenheit des Kerns, der Fortsätze v. s. w. unzweifelhaft als Nervenzellen und boten im Speciellen die Charaltere der grossen unter dem Namen der Purkinje' 
schen Nervenzellen bekannten Formen dar, wie sie in der innersten Lage der grauen Cortical substanz der Kleinhirnwindungen an der Grenze der rostfarbenen Schicht vorkommen. Von den in der heterotopischen Masse bei der mikroskopiscben Untersuchung gefundenen grossen Nervenzellen sind auf der beigefügten Tafel II unter Fig. 8 und 9 zwei Exemplare bei circa 300 facher Vergrösserung abgebildet; dieselben stammen aus einem frisch untersuchten und mit möglichst indifferenten Medien behandelten Stückchen aus der Mitte der heterotopischen grauen Masse. Da die Zeichnung nicht zu dem speciellen Zweck gefertigt wurde, um die Verhältnisse der Fortsätze und ihrer Verästelungen zum Ausdruck zu bringen, so sind letztere der Raumersparniss wegen nicht mit abgebildet, zumal die Beschaffenheit des Zellenkörpers für sich allein hinreichend cbarakteristisch sein dürfte um ihre Natur als Nervenzellen erkennen zu lassen.

Die feinen Knötchen enthielten, wie die mikrochemische Reaction zeigte, lohlensauren Kalk und zeigten einen geschichteten Bau.

\section{Dritte Beobachtung.}

Von den beiden vorstehend mitgetheilten Fällen unterscheidet sich der dritte in pathologisch-anatomischer Beziehung hauptsäcblich dadurch, dass in diesem die in Rede stehende Anomalie nicht blos in einer, sondern in fast symmetrischer Anordnung in beiden Hemisphären vorbanden war, - in klinischer Beziehung aber dadurch, dass der Träger der fraglichen Anomalie nicht an Epilepsie, sondern an paralytischer Geisteskrankheit gelitten hatte.

In Betreff der ätiologischen Verhältnisse ist besonders hervorzuheben, dass die gesammte Constitution und der kräftige, proportionale Körperbau jeden Gedanken an eine etwa zu Grunde liegende Degenerescenz ansschliessen liess, auch Form und Capacität des Schädels eine durchaus normale nnd entwickelte war, dass dagegen der Träger desselben etwa im 47. Lebensjahre, ein Jahr yor Ausbruch der Geistesstörung, einen schweren Fall auf den Hinterkop f erlitten hatte.

Auch in diesem Falle befanden sicb die heterotopischen Einsprengungen jemlich genau an derselben Stelle des Markstammes der Hemisphären, an weleher sie in den beiden bereits mitgetheilten Fällen vorbanden waren. Dieselben präsentirten sich jedoch auf dem Durchschnitte nicht unter dem Bilde eines continuirlich zusammenhängenden grauen Streifens, sondern in Form von einzelnen nabe neben einander liegenden und eine Reihe bildenden schmalen Inseln. Das gegenseitige Lageverhältniss derselben lässt sich nicht besser veranscbaulichen, als durch den Hinweis auf das geographische Bild, welches auf der Karte die Gruppirung von sog. Däneninseln in einer im Grossen und Ganzen dem Küstensaume ziemlich paralleI verlaufenden Reihe darbietet; - eine Configuration, wie sie namentlich die Küsteninseln der Nordsee vielfach zeigen. Wie diese Inseln sich sofort als eine genetisch zusammengebörige Gruppe kennzeichnen, so ist es auch mit den innerhalb der Meduilarsubstanz der Kleinhirn-Hemispbären längs der Grenzschicht 
vorbandenen in einer Reibe gruppirten Inseln grauer Hirnsubstanz der Fall. Am stärksten ist die Analogie der Gruppirung in der rechten Hemisphäre ausgesprochen: Die bier vorhandenen fünf Inseln grauer Hirnsubstanz zeigten ganz dieselbe Gruppirung, dieselben Grössen-, Form- und Distance-Verhältnisse wie die bekannten fünf Inseln, von Norderney bis Wangeroog auf der geographischen Landkarte. Sie waren von der graten Substanz der Windungen durch eine etwa noch $1 \frac{1}{2}$ bis $2 \frac{1}{2} \mathrm{Mm}$. breite Schicht medullarer Hirnsubstanz getrennt, unter einander aber durch Zonen weisser Substanz die noch schmäler waren. Nach verschiedenen Richtungen geführte Durchschnittezeigten, dass ein Zusammenbang dieser grauen Inseln mit der grauen Corticalsubstander Wind ungen nirgends bestand.

In der linken Hemisphäre des kleinen Gebirns fanden sich nur drei Inseln grauer Hirnsubstanz in der weissen Substanz des Markstammes - gleichfalls in einer Reihe und durchschnittlich $2 \mathrm{Mm}$. enternt von der Grenze der Corticalsubstanz.

Die hier bezeichneten Abnormitäten fanden sich bei einem Seelootsen, Alex. Scbm. (H. R. 922), welcher von Natur aus kräftig und regelmăssig gebaut, und mit guten geistigen Anlagen versehen, bis etwa zu seinem 45. Lebensjahre im Allgemeinen sich stets einer guten und dauerhaften Gesundheit zu erfreuen gehabt hatte, bei dem dann aber im 46. Lebensjahre eine, zunächst hauptsächlich mit psychischen Symptomen (furibunder Tobsucht, Grössenwabn) debütirende, als paralytische Geisteskrankeit zu charakterisirende und nach naheza zwei Jahre langer Daver letal endende Geistesstörung zur Entwicklung gekommen war.

Die Eltern des p. Schm. sollen beide eine sehr gesunde Constitution gehabt haben: der Vater ist 72 Jahre alt an Aitersschwäche gestorben, die Mutter batte wie es in dem ärztlichen Bericbte heisst „eine robuste, kernig frische Natur." Aucb die Geschwister des Schm. sollen körperlich und geistig gesund and überhaupt in der Familie Zeichen einer erblichen Disposition zu geistigen Erkrankungen nicht vorhanden sein. Nachdem Schm. die Elementarschule und später auch die Navigationsschule besucht und die erforderlichen Examina bestanden, ist er practisch als Seemann thätig gewesen und hat u. A. mehrere Seefahrten mitgemacht. Er ist dann in den Königlichen Dienst als Seelootse eingetreten und als solcher etwa 10-11 Jahre lang in Function, wäbrend des dänischen Krieges auch an Bord eines Kriegsschiffes (Vineta) commandirt gewesen. Mit Ausnabme von früher überstandenen Variolojden und einer intercurrenten Halsaffection soll Schm. sonst nicht eigentlich krank gewesen sein, dagegen ein Jahr vor Ausbruch der geistigen Störung einen schweren Fall auf den Hinterkopf erlitten (1866) und auch in der letzten Zeit mannichfachen dienstlichen Aerger gehabt haben.

Die nächste Veranlassung zum Ausbruch der Seelenstörung gab die Erregung lebhafter Affecte, die sich an einen Unfall auf einer Fahrt anknüpften. Auf einer zur Feier einer Hochzeit unternommenen Fabrt (5. Febr. 1867) war in Folge irgend einer Gebrechlichkeit der Wagen aus dem Geleise gekommen, der Kutscher von seinem Sitze herabgefallen und die plötzlich eingetretene Gefahr eines unmittelbar drohenden jähen Hinabsturzes des Wagens vom hohen Damme nur durch schnelles Herabspringen vom Wagen und entschlossenes Dazwischentreten Seitens des p. Schm. verhütet worden. An diesen Vorfall, von welchem S. anf der Hoch- 
zeit mit ungewöbnlicher Exaltation zu erzählen nicbt müde wurde, knüpfte sich unmittelbar eine zusehends sich steigernde Aufregung. Die Aufregung wuchs schnell, es trat schon in den nächsten Tagen eine eigenthümliche Verworrenheit der Ideen und unverkennhare Spuren des charakteristischen paralytischen Grössenwahns hervor: Schın. wollte alle Häuser kaufen, Stück für Stück für 1 Sgr., Oelbilder malen und Alles blank machen, legte selbst Hand an, fing an seinen Stock zu poliren, desgleichen einen Hauklotz, bohrte Löcher durch die Füsse des Tisches u. dgl. m. Bereits am 7. März war die tobsüchtige Agitation so gestiegen, dass man es für gerathen fand, den p. Schm. in das Lazarett zu Danzig zu bringen. Dort erfuhr die Tobsucht weitere Steigerung, so dass Isolirzelle und Zwangsjacke nöthig wurden. Ebenso erfuhr der Grössenwahn eine weitere Entwicklung: Pat. behauptete, er könne Alles, stelle z. B. mit einem Kostenaufwande von $\frac{3}{4} \mathrm{Sgr}$. eine ächte Vergoldung des eisernen Bettstelles ber, könne in ein Paar Augenblicken die schönsten Oelgemälde herstellen, elfenbeinerne Bettgestelle anfertigen u. dgl. m.

Mit diesen Symptomen, welche in Verbindung mit einer gewissen, allerdings nicht stark ausgeprägten Modification der Spracbe, auf den paralytischen Charakter der Geistesstörung hinwiesen, wurde Patient am 13. April 1867 in die hiesige Irrenanstalt aufgenommen. Bei der Aufnahme zeigten sich an verschiedenen Stellen der Extremitäten Durchreibungen resp. Erosionen der Haut, welche in Folge der furibunden Agitation und der dieserbalb dem Pat. angelegten Bandagen entstanden zu sein schienen und von welchen aus sich insbesondere am rechten Arm in der Gegend des Ellenbogens eine heftige phlegmonöse resp. psendo-erysipelatöse Entzündung entwickelt batte, die im weiteren Verlaufe den ganzen Arm in Mitleidenschaft zog, schliesslich abscedirte, und unter Anwendung von Kataplasmen, Umschlägen und wiederholten Incisionen allmählich verheilte, - zugleich aber auch auf die Psychose (sei es nun als Krisis oder starke Ableitung) einen sichtlich günstigen Einfluss hatte, insofern mit Entwicklung und Abscedirung der Phlegmone eine ganz bedeutende und ausgesprochene Remission der Exaltation und der Ideenverwirrung eintrat. Nach einigen Schwankungen des Befindens trat schliesslich auch der Grössenwahn gänzlich zurück; Pat. benahm sich Monate lang durchaus ruhig, angemessen und vernünftig, so dass eine versuchswtise Zurücknabme des Pat. aus der Anstalt gestattet wurde. Der Wiedereintritt in die Kreise und Beziehungen seines früheren Lebens am Heimathsorte bewirkte aber sehr bald eine erneute Exaltation, die sich so schnell wieder bis zur Höhe des früheren Anfalls steigerte, dass Pat. bereits schon am 29. Mai 1868 in voller Tobsucht und mit intensivem Grössendelirium der Anstalt wieder zugeführt wurde. Jetzt zeigte sich auch die Sprache merkbarer afficirt, nehmlich etwas anstossend und zitternd.

Dieser zweite Anfall übertraf den ersten noch bei Weitem sowohl an Intensität als auch in Betreff der Dauer. Mitten im Verlaufe der Tobsucht trat am 7.Juni Nachmittags um 5 Uhr ein paralytischer (apoplectiformer) Anfall ein, in Folge dessen der Mand nach links verzogen erschien. Bemerkenswerth war, dass während der Tage vorher in den Delirien des Schm. die Vorstellungen von Blat und Feuer in ganz besonders auffallender und prägnanter Weise hervortraten. So erklärte er z. B. die Wände seiner Zelle (in Wirklichkeit mit einer mattgrauen Oelfarbe angestrichen) seien blutroth, das was er ausspucke, sei Blut (in Wirklich- 
keik gewöbulicher Speichel). Auch nach dem paralytischen Anfalle, von welchem Pat. sich übrigens sebr schnell wieder erholte, machte sich noch eine geraume Zeit lang (so lange nehmlich der congestive Zustand vorherrschte) eine auffallende Präponderanz der Vorstellungen von Feuer, Blut und Brand geltend: Pat. batte verschiedene blutige und feurige Visionen, sah Spiritus in seiner Zelle aufflammen und sogar sein eigenes Bett brennen, in dem er lag; behauptete, es brenne über und unter ihm und sogar in seinem eigenen Leibe u. s. w. Im A ugust localisirte Variolois. Im weiteren Verlaufe machte sich unter Fortdauer der Tobsucht und des Grössenwahns einige Abmagerung und deutlicher hervortretende Symptome einer allgemeinen unvollkommenen allmäblich fortschreitenden $\mathrm{L} a ̈ \mathrm{~h} \mathrm{mu}$ ug bemerklich, desgleichen Geruchshallucinationen. Auch Gehörshallucinationen scheinen nicht gefehlt zu baben, wenngleich sie nicht so deutlich und ausgeprägt hervortraten wie die Gesichtsballucinationen, und mehr den Charakter innerlich laut werdender Gedanken hatten, mit welchen sich Pat. in lautem Monologe unterhielt.

Der Tod erfolgte ziemlich plötzlich nach eidem kurzen paralytischen (apoplectiformen) Anfalle, zu welchem sich, nachdem das Bewusstsein bereits wieder zurückgekehrt war, Lungenö dem hinzugesellt batte.

Die Section der Leiche (20 St. n. d. T.) ergab im Wesentlichen folgende Befunde: Magerer Körper, an der Dorsalseite einige durchgelegene aber wieder yerheilte Steilen.

$\mathrm{Sch}$ ädel gross, $19,9 \mathrm{Cm}$. lang, $16 \mathrm{Cm}$. breit, von symmetrischer Form und ziemlich schwerem Gewicht, vorwiegend ans compacter Knochensubstanz bestehend. Auf der inneren Oberfläche der Dura mater befindet sich sowobl an der Basis als auch an dem, dem Schädeldache entsprechenden Theile eine allerdings nur sebr dünne und zarte, stellenweise nur schwach angedeatete Pseudomembran; stärker ausgeprägt erscheint letztere auf beiden seiten der Falx. Weiche Häute auf der Convexität getrübt, verdickt und ödematös. Seitenventrikel etwas erweitert, strotzend mit wasserklarem Serum erfüllt, Ependym verdickt und granulirt. Graue Substanz der Thalam. optic. fablgelblich entfärbt. Temporal-Lappen etwas erweicht. Corticalsubstanz der Windungen an der convexen 0 berfläche der beiden Hemisphären des grossen Gehirns fahlgelblich entfärbt, in den Windungen der Frontal-Lappen von trübem Aussehen. Medullarsubstanz in den hinteren Lappen auf der Schnittläche stärker einsinkend als in den vorderen Lappen. Vordere innere Partie der Pyramiden von graner Färbung. Striae acusticae stark entwickelt, rechts stärker als links. Rückenmark: Graue Substanz etwas atrophisch, auf der Schnittfläche ziemlich stark einsinkend; weisse Substanz der hinteren und seitlichen Stränge etwas streifig; im unteren Brustmarke vorwiegend der rechte Seitenstrang afficirt. Am Cerebellum zeigen die weichen Häute nur eine geringe Trübung. Beide Langen stark ödematös. Herz ohne Fettauflagerung auf der Oberlläche.

Die mikroskopische Untersuchung der in dem Markstamme der Kleinhirnhemisphäre vorbandenen inselförmigen grauen Massen liess in derselben eine Anzabl zelliger Elemente erkennen, welche den in den beiden anderen vorstehend mitgetheilten Fällen vorgefundenen durchaus analog waren und als unzeifel h hafte Nervenzellen angesprochen werden mussten. 
Was nun die klinische Bedeutung der Heterotopie graver Hirnsubstanz im Markstamme des kJeinen Gehirns anbetrifft, so würde es bei dem Dunkel, in welchem wir uns in Betreff der Functionen gerade dieses Organs zur Zeit noch befinden, gewagt erscheinen, wollte man auch nur eine Hypothese aufstellen äber die durch die beregte Anomalie etwa bedingten Functionsstörungen. Nur insoweit darf man vielleicht von der Anwesenheit solcher Structur-Anomalien auf eine gewisse Prädisposition zu gewissen Functionsstörungen schliessen als die beregte Heterotopie eine von der Norm abweichende Entwickelung des in Rede stehenden Centralorgans überhaupt bekundet, gleichsam ein makroskopisch sichtbares Merkzeichen darstellt, welches auf früher bei der Entwickelung des Gehirns orer vielleich $t$ auch in späteren Lebensepochen stattgehabte anomale Vorgänge hinweiset, die nicht blos die makroskopisch sichtbaren, sondern wahrscheinlich auch feinere, der directen Wahrnehmung weniger zugängliche Structur-Anomalien bedingt haben. Ausserdem ist durch den, wenigstens in den zwei zuerst mitgetheilten Fällen, in Betracht kommenden Umstand, dass die Anomalie nur in der einen (linken) Hemisphäre vorhanden war, ein Moment gegeben, welches für das richtige Gleichgewicht der Functionen der beiden correspondirenden Hemisphären wohl nicht ganz gleichgültig sein dürfte. Da die in beiden Fällen vorhandene Epilepsie in Bezug auf die sich später entwickelnde Geistesstörung als das primäre Leiden erscheint, so möge hier beiläufig auch an die Experimente Nothnagel's ${ }^{1}$ ) erinnert sein, aus denen hervorzugehen scheint, dass sich in der Brücke - also in einem Organe, in welches Fasern des Markstammes des kleinen Gehirns einstrahlen ein Centralapparat befindet, durch dessen Vermittlung „allgemeine epileptiforme Convulsionen" bewirlt werden.

In $p$ athog enetischer Beziehung erscheint es, - da für die Annahme, dass die in Rede stehende Heterotopie erst während des Lebens entstanden sei, alle Anhaltspunkte feblen, namentlich in dem zuerst mitgetheilten Falle - zunächst gerechtfertigt die fragliche Abnormitït als eine angeborene resp. bald nach der Geburt zu Stande gekommene zu betrachten. Für die Annahme, dass dieselbe schon bei der Geburt vorhanden oder doch wenigstens in

1) Die Entstehung allgemeiner Convulsionen vom pons und von der Medulla oblongata aus. Dieses Archiv XLIV. 1-12. 
ihrem Keime präformirt gewesen sei, also auf irgend einer Anomalie der intra-uterinen Entwicklung beruht habe, lässt sich der Umstand anführen, dass diese Entwicklung in einem, wenn nicht bereits in der Involution begriffenen, doch jedenfalls dicht an der Schwelle des Stadiums der geschlechtlichen Decrepidität st ehenden Organismus stattgefunden hat. - In den beiden anderen Fällen waren positive Momente, welche für eine solche auf mangelhafter Entwicklung beruhende Pathogenese hätten sprechen können, nicht vorbanden; vielmehr zeigten in diesen beiden Fällen die betreffenden Individuen eine besonders kräftige und proportionale Bildung des Körpers ônd namentlich auch günstige Schädelverhältnisse - desgleichen bestand in Bezug auf dieselben auch keinerlei nachweisbare familiäre Disposition zu Geistes- und Nervenkrankheiten, Apoplexien u. dgl. $O b$ jedoch die durch äussere mechanische Gewalt herbeigeführten Insulte, welche Hirn und Scbädel in diesen beiden Fällen erlitten und in Folge deren sich entzündliche Prozesse an der Hirnoberfläche entwickelt hatten in irgend einem causalen Zusammenhange mit der in Rede stehenden Heterotopie stehen, dürfte sich z. Z. nicht entscheiden lassen und mag die bestimmtere Beantwortung dieser Frage etwaigen weiteren Beobachtungen vorbehalten bleiben.

Auf Grund meiner in Vorstehendem mitgetheilten Erfahrung möchte ich die Vermuthung aussprechen, dass Anomalien der gedachten Art gewiss öfter zur Beobachtung kommen werden, sobald nur erst dieser Punkt Gegenstand grösserer Aufmerksamkeit geworden sein wird. Der Umstand wenigstens, dass ich während eines Zeitraums von drei Jahren drei Mal die in Rede stehende Anomalie vorgefunden habe, dürfte doch einigermaassen dafür sprechen, dass auch im kleinen Gehirn Heterotopien grauer Hirnsubstanz öfter vorkommen, als man bei dem bisherigen Mangel solcher Beobachtungen anzunehmen geneigt gewesen ist. Ich möchte hierbei daran erinnern, dass auch in Betreff der im Innern der Medullarsubstanz des grossen Gehirns vorkommenden Heterotopien die Zahl der beobachteten Fälle sich zusehends vermehrt hat; denn seit Veröffentlichung ${ }^{1}$ ) meiner ersten Beobachtung dieser Art im Jabre 1864 und seit der

7) Allgemeine Zeitschrift f. Psychiatrie. 1864. Bd. XXI. S. 481 . 
Mittheilung einer sehr bemerkenswerthen Beobachtung von Virchow anf der Naturforscherversammlung zu Hannover ${ }^{1}$ ) sind sowohl von mir selbst ${ }^{2}{ }^{3}$ ) als auch von anderen Autoren: C. K. Hoffmann in Meerenberg ${ }^{4}$ ), Gottlieb Merkel in Nürnberg ${ }^{5}$ ) und B. Wicke in Göttingen ${ }^{6}$ ) analoge Fälle beobachtet worden.

\title{
Erklärung der Abbildungen.
}

\author{
Taf. II. Fig. 3, 4, 8 u. 9.
}

Fig. 3. Photographische Abbildung eines vertical $b_{5}$ und parallel der Längsaxe der Med. obl. geführten Durchschnitts durch die linke Hemisphäre des kleinen Gehirns. U Unterer Rand, 0 obere Partie des Hemisphärenabschnıtts, von welcher jedoch ein Theil ganz entfernt ist. M Markstamm des Arbor vitae. Innerhalb der weissen Substanz derselben, nahe der graven Corticalschicht der Windungen an der unteren Seite U (hinterer Unterlappen) ein grauer Streifen heterotopischer Hirnsubstanz, von h bis $g$ sich erstreckend; bei b schwächste, bei g stärkste Entwicklung dieses Streifens. d d Lumina durchschnittener Gefässe. Ik k Nadeln, mit welchen das Präparat während der photographischen Aufnahme befestigt war.

Fig. 4. Ganglienzellen aus der in Fig. 3 dargestellten, im Markstamm des kleinen Gehirns vorhandenen heterotopischen grauen Hirnsubstanz: b, c, d grosse Nervenzellen mit Fortsätzea, deutlichem Kern und Kernkörperchen; a, a, a Nervenzellen ohne Fortsätze und mit weniger deutlich erkennbarem Kern und Kernkörperchen.

Fig. 8 u. 9. Zwei Ganglienzellen aus den in dem Markstamme der linken Kleinhirnhemisphäre (vgl. S. 86) heterotopisch eingesprengten grauen Inseln bei 300 facher Vergrösserung (vgl. S. 90). Von den Fortsätzen sind nur die Anfangstheile gezeichnet, die weiteren Verzweigungen aber weggelassen.

1) Tageblatt der 40. Versamml. deutscher Naturf. u. Aerzte zu Hannover i. J. 1865. No. 3. S. 38. - Virchow's Archiv. XXXVIII. 138.

$\left.{ }^{2}\right)$ Dieses Archiv. XXXVII. 567 .

3) Dieses Archiv. L. 297.

4) Nederlandsch Tijdschrift v. Geneeskunde. 5. Jaarg., 5. Afd. - Henle u. Pfe uffer, Zeitschr. f. rat. Med. Bd. XXXIV. Heft 1.

5) Dieses Archiv XXXVIII. 433.

6) Deutsche Klinik 1869. No. 19 u. 20. S. 175 u. 185. 